\title{
Automation in cardiology
}

\author{
Leon Resnekov ${ }^{1}$ \\ From the Department of Medicine (Cardiology), University of Chicago, \\ 950 East 59th Street, Chicago, Illinois 60637, U.S.A.
}

The basic principles of analogue and digital computers are reviewed and their application is described for the beat-to-beat analysis of the electrocardiogram using the AZTEC data reduction programme, for on-line haemodynamic analyses in the cardiac catheterization laboratory, coronary care unit, or postoperative ward, and for the clinical history and physical examination of the patient. The role of the computer in the management of cardiovascular disease is defined and its superiority in the collection and manipulation of complex data is emphasized. Its use in sparing staff the tedium of routine analyses of data will amply repay the initial cost of its installation.

'Medicine is a science of uncertainty and an art of probability.'

\section{Sir William OsLeR}

Great strides have been made during the last decade in the application of the computer to biological problems, and cardiology has shared in the continued development of these new techniques. This is hardly surprising, for there are few areas in clinical investigation which embrace so many basic principles as are used during the measurements of intravascular pressure, flow, myocardial function, heart chamber size, and the electrocardiogram. The continued improvement and refinement of cardiac investigation has demanded, and obtained, sophisticated electronic systems, but one hour of such investigation may be followed by 80 hours of data processing to extract all the information being recorded (Taylor, 1966). Without a parallel development in computer technology and systems-engineering science the relative ease with which very complex circulatory data can now be obtained will be largely wasted, for the great increase in the production of data itself restricts further expansion unless methods are available for automatic measurement and analysis.

This paper reviews recent developments in the application of computer techniques to three areas with immediate relevance to the patient with cardiovascular disease - namely, his history and physical examination, the

${ }^{1}$ Supported in part by grants from the University of Chicago Myocardial Infarction Research Unit and the Chicago Heart Association. electrocardiographic analysis, and on-line pressure and flow determination.

\section{Historical review}

Arriving at a medical diagnosis on the basis of a logical and scientific process has always suffered from two inherent faults: an ignorance of the number of diseases producing similar signs and symptoms, and an inability on the part of the physician to remember all the diagnostic information he needs for an accurate diagnosis. Some device is needed by which the physician can be helped and both deficiencies repaired. Earliest aids included devices resembling slide rules by which a physician could match combinations of signs and symptoms and thereby choose the most likely diagnosis out of a possible number exceeding 300 (Nash, I954, I960). Though interesting, these could hardly be useful as an overall solution to the problem, and thereafter mechanical methods were introduced involving card and needle systems (Lipkin and Hardy, 1958) but no probability relationship was possible using these approaches. Ledley and Lusted (1959) proposed that medical diagnosis be considered as logic processes and as a conditioned probability according to Baye's theorem, and Warner et al. (196I) were able to use this approach successfully in the diagnosis of congenital heart disease. The increasing availability of digital computers has provided a better method for collecting and coding medical information, and at the same time the essential newer mathematical approaches for dealing with biological problems have been developed (Zadeh, 1962). 
While the whole field of computer application to biological problems is rapidly expanding, it is important to try to maintain an overall view of the field as a whole and its application to medical problems in particular. There are many classifications of the functions of an electronic computer and a convenient list has been proposed by Schmitt (1962) as follows:

I) Transduce - to translate a process between one physical representation of data and another.

2) Transpond - to guide the experiment in terms of instant appraisal according to results already being computed.

3) Sort and assemble.

4) Store and retrieve.

5) Compute.

6) Simulate and synthesize.

7) Display.

Although it is true that a physician might require several years of study to be expert in all aspects of the computer, much less effort is needed to understand its basic principles, including acquiring the necessary skill in programming.

Data for processing are often presented as digital phenomena in which a finite number of symbols or characters are used. In contrast, analogue data involve no explicit symbol or language but a representation of the data as a magnitude of physical quantity - for example, length or voltage. Most instruments used in clinical measurement display an output as an analogue signal, whereas direct measurements - for example, measuring the blood pressure - provide digital data. In the same way, calculations may be made either in an analogue or digital form. Analogue computers provide great precision but at the cost of increasing technical difficulty. Digital computers increase their precision by increasing the number of significant figures used; doubling these numbers will roughly double the size and therefore the cost of the installation. There are many problems in clinical medicine for which analogue computers are inadequate, although if only a modest degree of accuracy is required an analogue computer is a relatively cheap and ideal instrument. It is important, therefore, for the physician to define clearly the degree of accuracy of his experiment, for if only an accuracy of one per cent is possible, performing calculations based on these data to any greater degree of precision is not only unnecessarily costly but is also a form of selfdeception.

Whereas both digital and analogue computers require skilled programmers, the digital computer programmer works with the input and output devices of the machine. In contrast the analogue computer is programmed by interconnecting various parts of its circuit, and the output is a voltage which may be displayed in a variety of ways - for example, a meter, an $x-y$ plotter, or an oscillograph.

The main advantage of the digital computer is its high degree of versatility. Provided that a problem can be put in a numerical or symbol form, it will be solved as long as the computer has enough time to complete the solution and enough memory to make it able to do so. The analogue computer is more limited, but has a particular role to play in solving differential equations, including the simultaneous non-linear variety. These computers are often designed and built for the solution of a particular problem, whereas digital computers are more general purpose instruments. As would be expected, the analogue computer is much less costly than the digital instrument.

The development of specialized myocardial infarction research units (Bondurant, 1969; Frommer, 1968), which have come about largely owing to the foresight of the late $\mathrm{Dr}$. Robert P. Grant, has provided a fruitful setting for patient research, including the clinical presentation, the circulatory abnormalities present, and the investigation of the effects of the cardiac lesion on each of the body's systems. In addition it has been possible to devote considerable resources in terms of manpower and funds to the development of computer techniques for the better handling and understanding of the large amounts of data collected. The section which follows describes in outline computer techniques which were developed as part of the Myocardial Infarction Research Unit of the University of Chicago.

\section{Electrocardiographic analysis}

Perhaps in no other area is the immediate recognition of rhythm disturbances of greater practical importance than in the patient with acute myocardial infarction. The majority of deaths occurring in the acute phase of the disease relate to the development of cardiac rhythm disturbances; ventricular extrasystoles which under different circumstances may have less dire consequences may now precipitate ventricular tachycardia, fibrillation, and death (Meltzer et al., 1964). The establishment of coronary care units (Day, 1963; Brown et al., 1963) has resulted in a significant reduction in mortality after acute myocardial infarction largely owing to the prevention, recognition, and early treatment of cardiac dysrhythmias; the management of cardiogenic shock, however, still remains a 


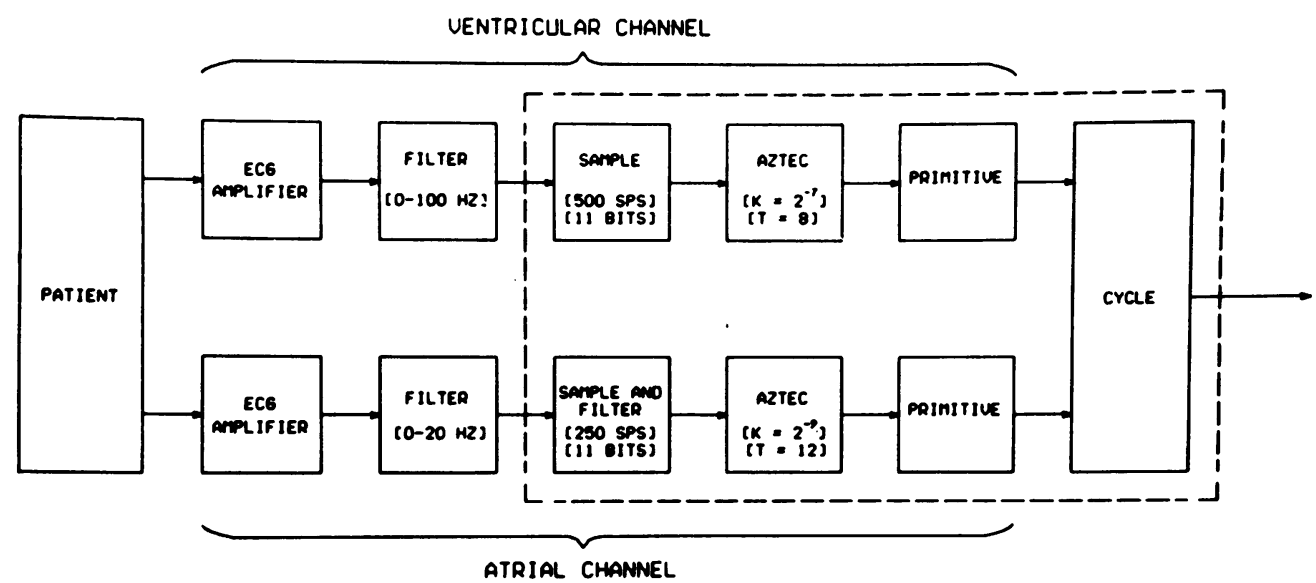

FIG. I Block diagram showing steps for processing, condensing, and analysing the electrocardiogram. The blocks within the dashed lines are carried out by a digital computer programme (PDP-8I).

therapeutic challenge. For the efficient recognition of potentially dangerous rhythm disturbances some system of continuous monitoring of the electrocardiogram is desirable. The more conventional forms of monitoring the analogue signal, with or without additional rate alarm devices, while of undoubted use, have shortcomings, and furthermore can give little help in the recognition of complicated rhythm disturbances. In addition the editing and storing of continuous electrocardiographic records, recorded on either tape or paper, become a formidable undertaking. Processing a digital conversion of the electrocardiogram on-line by the computer is an attractive alternative approach. It is already well recognized

FIG. 2 Reconstructions of the output of (a) the sample, (b) the AZTEC processor for the ventricular channel, and $(c)$ the line symbols produced by the processor.
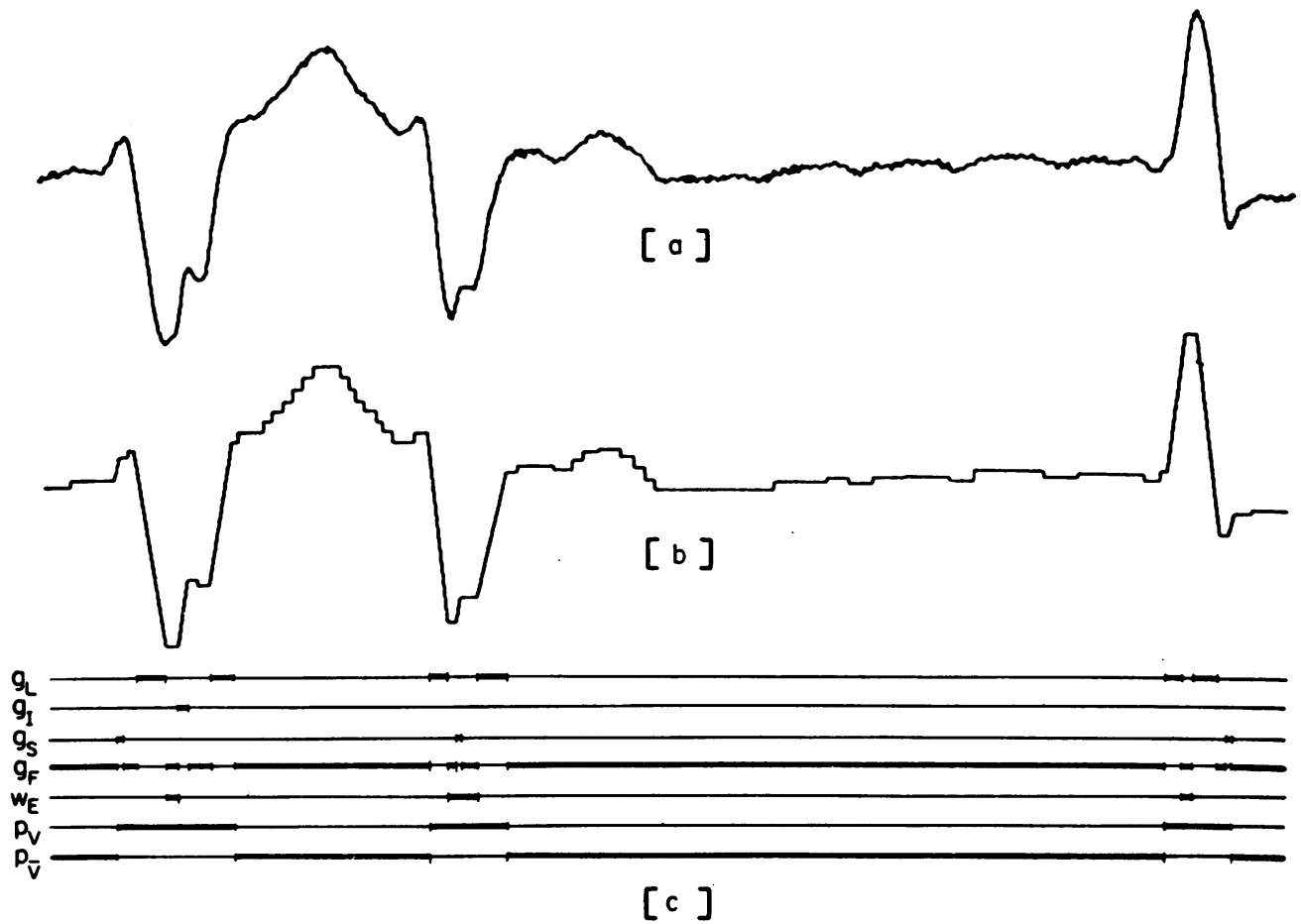
that the patient with acute myocardial infarction frequently develops transient sudden and dramatic changes in his electrocardiogram. Any really useful system must be capable of recognizing these events and interpreting them correctly, as well as differentiating baseline fluctuations, muscle artefacts, and electrode or electronic noise. Analogue to digital conversion of the electrocardiograph signal is usually undertaken at rates between 100 and 500 samples per second, but this is impractical for continuous on-line analysis when a form of process that keeps up with the raw input data is needed. Programmes are now available for eliminating redundancy in the electrocardiograph signal yet maintaining diagnostic accuracy (Cox et al., 1969). In this programme unimportant detail is eliminated by a series of processors (Fig. I) each producing a reduction in data rate of a factor greater than two, and the system of processing, condensing, and coding has been called AZTEC. This is achieved by converting the electrocardiograph signal into a set of lines using a linear interpolator, each of which represents an average voltage and duration and uses only two 12-bit words in memory (Fig. 2). A further step condenses sets of lines into slopes, and each slope requires only two words in memory. The overall result is an ordered set of lines and slopes with a response to $50 \mathrm{~Hz}$ available for detailed analysis, and the data rate is 20 word pairs per second. As the electrocardiograph signal is not prefiltered, muscle noise and baseline drift are recognized by a separate programme which classifies and displays the signal in several ways: (i) not analysable, (ii) noisy but adequate for limited analysis, or (iii) quiet, when complete analysis is possible. The programme recognizes the QRS complex, codes it as to its shape, measures its duration and the length of the previous cycle, and also takes note of the repolarization process so as to avoid confusion with other parts of the signal. A small fast response digital computer (PDP-8I) is used in the analysis and storage of data.

The problem of identifying the electrical signs of atrial depolarization is similar to, but not identical with, the recognition of the ventricular complex. The $\mathrm{P}$ wave recorded at the body's surface is smaller in amplitude and subject to distortion by noise, but on the other hand the shape of the $P$ wave is generally less variable than that of the QRS complex, and in our original system a separate AZTEC processor was used in the atrial channel operating in a manner similar to that in the ventricular channel (Fig. I). The programme worked extremely well and the normal electrocardio-

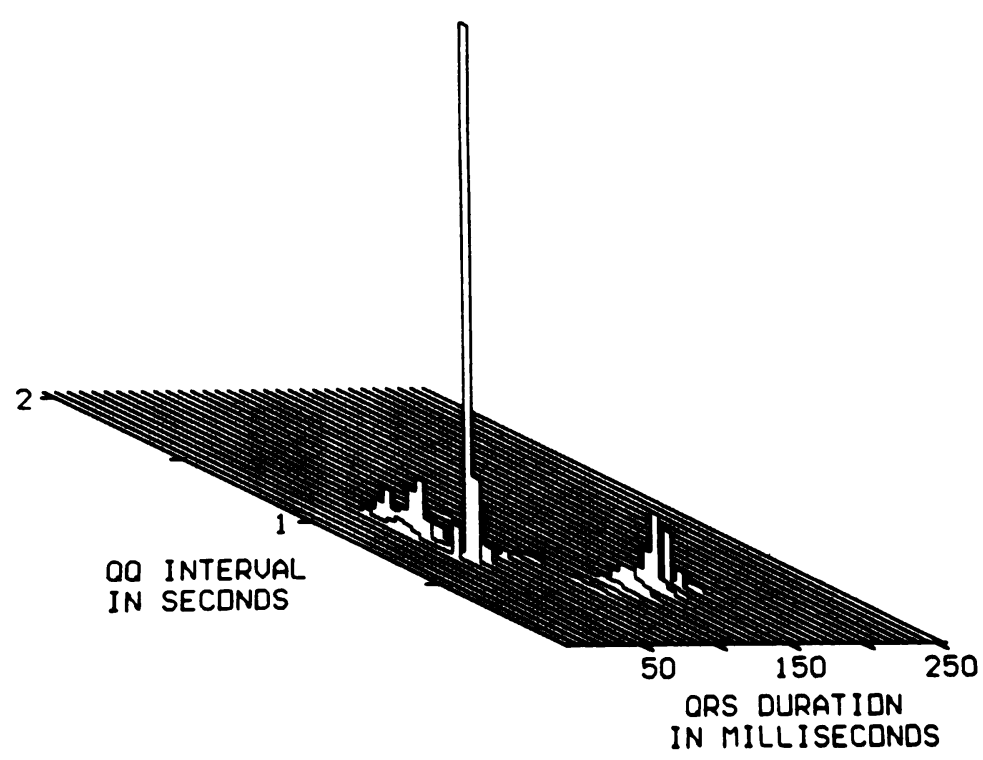

FIG. 3 Two-dimensional histogram of $Q Q$ intervals and $Q R S$ durations. Normal sinus rhythm is shown. The beats at the lower right have a reduced $Q Q$ interval and a widened $Q R S$ and are bigeminal ventricular extrasystoles.

graph and common rhythm disturbances were consistently recognized and coded. Complex dysrhythmias, however, where accurate $\mathbf{P}$ wave recognition is mandatory, were less consistently recognized, and the original programme has now been successfully modified to accept a right atrial intracavitary lead with excellent results.

FIG. 4 Two-dimensional histogram from a patient with atrial fibrillation. There is great variability in the $Q Q$ interval, but the $Q R S$ duration is relatively stable. The small peak at the lower right is a set of beats conducted aberrantly.

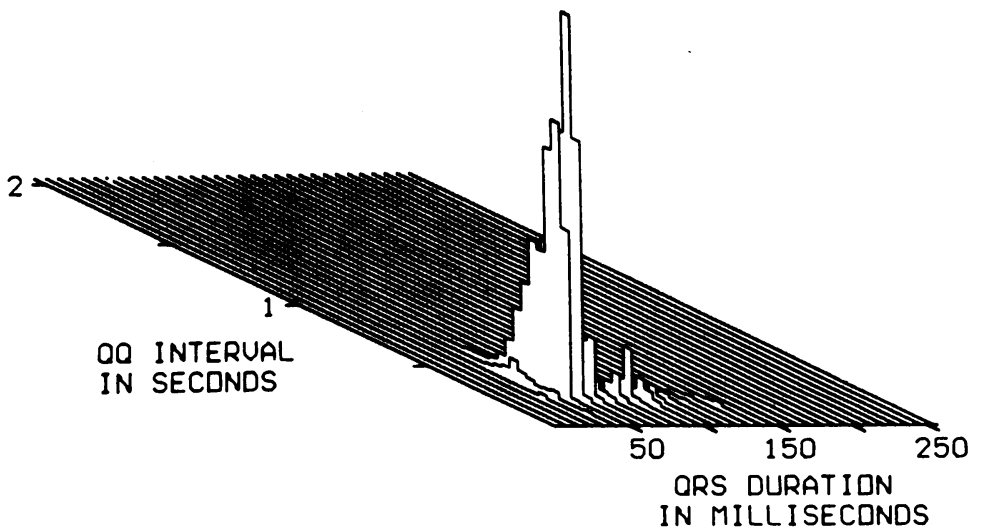


After these preliminary measurements, each individual cycle is diagnosed. The basic rhythm and premature beats, atrial, junctional, or ventricular, are recognized at this stage. Additional measurements then follow, and the shape of the ST segment, and the direction and height of the $\mathrm{T}$ wave and QT interval are recognized and coded.

Extensive testing of the programme in practice indicates that at no stage has important information crucial to the correct clinical diagnosis been compromised. The results can be displayed using an oscilloscope or teleprinter, or alternatively histograms may be derived from the cycle-processor output. In such a two-dimensional display the QRS duration is plotted horizontally with the QQ intervals in perspective. An example is shown in Fig. 3, in which the electrocardiogram of a patient in normal sinus rhythm interrupted by ventricular extrasystoles has been analysed. The extrasystoles are shown at the lower right of the figure, indicating a reduced $\mathrm{QQ}$ time and a widened QRS complex. In Fig. 4 a similar plot is shown for atrial fibrillation. There is now considerable variability in the $Q Q$ intervals and in the stability of the QRS duration. The small secondary peaks at the lower right form a set of aberrantly conducted beats.

\section{Haemodynamic data}

On-line haemodynamic measurement is a useful facility in the cardiac catheterization laboratory for the almost instantaneous analysis and display of data and in the coronary care unit or postoperative ward for the management of acutely ill patients. Several programmes are now available by which such data can be collected and analysed. We use a Fortran-I I programme (Blackstone, 1967) for the computer handling of haemodynamic data, including on-line analysis of pressure and flow. Certain events in the mechanical cardiac cycle are recognized and manipulated mathematically to provide useful information about each cycle and to average these events over the whole period of recording. Data are collected on multichannel FM analogue tape and edited, and selected parts are converted to binary data using a specially prepared analogue-to-digital conversion programme. Calculations made from the central arterial pressure wave allow accurate estimation of stroke volume, heart rate, cardiac output, mean pressure, duration of systole, peripheral resistance, and systolic and diastolic pressures, all on a beat-by-beat basis by equations and derivations which have already been published (Warner, I965) and the validity of the methods proved against direct

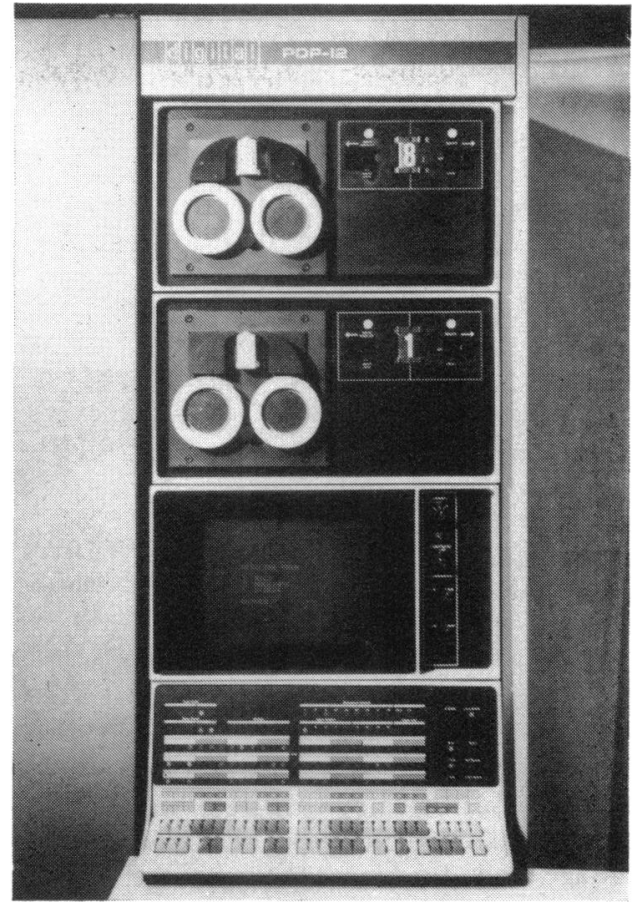

FIG. 5 PDP-12 computer being used for the history and physical examination programme.

measurements both in dogs and man. The basic time-sharing computer system can readily be extended so that no fewer than 12 user-programmes can be in core memory at any one time (Warner, 1969). The indirect estimation of cardiac output by calculating stroke volume from the aortic pressure wave curve (Warner et al., 1953) has been a great advance. The output of the pressure transducer is sampled at 100-200 times per second and led to the computer whose pattern recognition programme identifies the onset and the end of each systole. The stroke volume is obtained from the definite time integrals of pressure with respect to time, and the system can be calibrated if desired against an independent measurement of cardiac output during the steady state. Continuous monitoring of the stroke volume is thereby possible for an indefinite period, though subsequent changes in the peripheral vascular resistance may require further calibration of the method against an independent measurement of cardiac output. Comparison of cardiac output as derived from stroke volume measurements made in this way against indicator dilution techniques indicates a high degree of correlation (Warner, 1965).

In a similar fashion, pulmonary arterial, 
ventricular, and atrial pressure pulses can be handled and analysed by the computer and the first and second derivatives of the ventricular pressure pulses obtained on-line and the results displayed on a digital meter, teleprinter, or oscillograph.

\section{History and physical examination}

Despite the undoubted importance of accurate history taking and physical examination, very little research has been undertaken into the methods of obtaining a history or the reliability of physical signs collected in this way. In the time-honoured system, medical histories and physical examinations are recorded longhand and are therefore unstandardized and often incomplete or written illegibly so that the use to which such information can subsequently be put for patient care or clinical research is limited. Several medical questionnaire systems have already been popularized (Brodman et al., 1949, 1959; Collen et al., 1964). The early systems, however, were designed to conform to a dataprocessing card as an input medium. Of more use is a questionnaire designed primarily for physician diagnosis which has the same flexibility in history taking as the physician himself, but surpasses him in available detail, consistency, legibility, and economy. Such a system was described by Slack et al. (1966) and consists of a conversational programme for use with a rapid computer of the LINC type. In general, the programme operates by questions and statements being displayed on a cathode ray screen and responses being made on a computer keyboard. Printed summaries of the patient's history and the physician's physical examination are generated by teletype at the end of each interview. All responses are saved on magnetic tape for future processing by the computer, both for patient care and for clinical research. The computer we now employ (PDP-I2) is a small, general purpose high-speed digital instrument especially designed for collection of data directly by the computer and for processing data as fast as it is generated. Two magnetic tape units provide for storage and retrieval, and the cathode ray oscilloscope, which resembles a television screen, is capable of displaying easily readable statements (Fig. 5). The computer-based questionnaire provides four possible responses: (i) yes, (ii) no, (iii) don't know, and (iv) don't understand. If the response to a question is ' $y e s$ ', a specific series of qualifying questions follows; if 'no', the qualifying questions are skipped and another general question is asked. When the answers to certain questions are not known, or if the questions are not understood, the questions are qualified with varying simplified terminology.

Using this basic programme, we have modified the system to be suitable for histories from patients admitted to a cardiology ward and to the coronary care unit, and also to handle the physical examination, the day-today follow-up notes, the electrocardiographic analysis, and other special investigations performed. The computer-based sheets serve as the clinical notes for that patient, and a permanent hard copy teleprint summary is provided. Perhaps the greatest use of the system is that it is ideally suited for the collection of data to be statistically analysed by the computer for research purposes at some future date, for each patient in essence is asked and responds to identical questions, the physical examination is complete in every patient, and all the investigations are entered sequentially.

\section{Discussion}

Computer techniques are only now becoming accepted as useful tools in clinical medicine, although their place has been accepted in engineering and the biological sciences for many years. Their particular role in the clinical cardiological situation is to exploit fully the abundance and complexity of modern circulatory investigation, for these devices have a facility for collecting and analysing data at a speed which is unrivalled. As our investigational methods become more complex, some means is urgently needed for reducing the personnel time required at present to analyse all the data recorded. Furthermore, the correct mathematical and statistical handling of such data is essential for accurate patient diagnosis and management. The computer therefore provides the clinical investigator with a versatile instrument whose full potential is limited only by the conceptual approach of the programmer rather than by any inherent deficiency of the instrument. As already emphasized, the use of the computer in cardiovascular diagnosis is not limited only to the cardiac catheterization laboratory but in addition it has an important role in the electrocardiographic analysis as well as in the long-term monitoring of the acutely ill patient in the coronary care unit or postoperative ward. Even in the more mundane setting of the clinical ward it will maintain a degree of precision previously lacking in history taking, physical examination, and follow-up of the patient.

The initial outlay for the computerization 
of clinical and investigational data is not inconsiderable, so that it might well be asked whether the programmes at present available and the apparatus now in use are capable of providing the precision we now lack. Previous studies of observer variation in the analysis of physiological signals show clearly that computer analysis is a significant advance (Caceres, 1963; Dobrow et al., 1964). Studies of these kinds, furthermore, emphasize that the computer also helps to establish standards of uniformity in the interpretation of collected data and standards of comparison for studies involving large groups of patients.

Computer programmes have also been devised for other aspects of cardiovascular investigation - namely the phonocardiogram (Coleman and Caceres, 1963). Using this programme, the duration of systolic and diastolic intervals, the relative intensities and time intervals of the various valve components, and the duration and relative intensity and frequency characteristics of murmurs during systole and diastole can be obtained and a useful phonocardiographic diagnosis arrived at. Other workers have attempted the computer analysis of the phonocardiogram using the energy level of sounds (Gerbarg et al., 1962), and in addition analogue circuits have been established to identify heart murmurs (Konecci, 1964).

Although there is no technical limit to the computer's clinical use, it is necessary for the investigator to define precisely his data in terms which can be understood and analysed. This is relatively easy when dealing with pressure, flow, and electrocardiographic data, but becomes increasingly more difficult for history taking and the physical examination. Any critical analysis of currently employed methods for recording these important items - namely the hospital chart - reveals its complete inadequacy for considering the patient's problem in detail or as a prospective document to be used later in clinical research (Weid, 1967). There are now available several computer-based history-examination systems, one of which is described in the earlier part of this paper. While the computer programme is satisfactory from the point of view of the physician, it is equally important to consider the patient's reaction to this form of medical interview. Such a study has now been completed (Slack and Van Cura, 1968) in which 275 patients responded to questions displayed on a cathode ray screen by using a computer keyboard. Most indicated their approval of this novel form of interview, and furthermore there was no correlation between the social and economic background of the patient and his opinion of the computer system. Further experience has confirmed the initial favourable reaction, on the part of both the patient and the physician, but perhaps its greatest asset is its thoroughness in collecting important historical information and recording a complete physical examination, making subsequent analyses for clinical research so much easier.

As could be predicted, the electrocardiogram was investigated early by computer scientists in an attempt to provide a detailed and accurate analysis of the information contained. Several programmes have been worked out completely after the initial studies, including the AZTEC system described earlier (Cox et al., 1969). Others have preferred an analysis of special lead systems, and the corrected orthogonal three-lead electrocardiogram system has been recommended (Pipberger, 1965). The application of dataprocessing systems clearly makes it highly desirable that a reduction in the number of leads is considered, provided that no useful information is lost in this way. Further analysis of the three-lead orthogonal record by digital computer is now developed to the point where it can provide accurate diagnoses in routine use (Pipberger et al., 1963; Pipberger and Stallmann, 1964). Others have continued to use the standard 12-lead electrocardiogram for routine computer analysis (Caceres, 1965). The electrocardiogram can be recorded on magnetic tape at the patient's bedside or sent by telephone to the computer laboratory for recording and analysis (Cooper and Caceres, 1964). Though in published trials the proportion of computer measurements within the level of useful clinical interpretation is as high as 97 per cent (Caceres et al., 1964), the most common errors it makes are incorrect determination of electrocardiogram wave onset or end. While the $T$-wave recognition is now less of a problem, many workers feel that the $P$-wave is best recognized using a right atrial intracavitary lead. When the AZTEC system was put to critical analysis (H. Fozzard, 1969, personal communication), obvious missed beats occurred in about one per cent of the total number of QRS cycles analysed. These were almost entirely the result of poorly adjusted amplifier gain resulting in the signal being outside the range of the A-D converter. In addition, when patients were studied for stability of the analysis in the presence of a noisy record, about 0.6 per cent of all cycles analysed were interrupted. Obtaining clean signals will materially help this problem, though, even when the record is known to be noisy, in practice only about one 
cycle in every 200 cannot be analysed by the computer.

It is clear, therefore, that the computer is both powerful and useful in cardiovascular diagnosis. Provided it can be given analysable data, there is no reason why all the currently employed cardiovascular techniques cannot be handled in this way, including the phonocardiogram, vectorcardiogram, and angiocardiogram. Computer applications are more easily introduced when system engineers and medical scientists are working together as a team. Neglect of the one or the other inevitably leads to frustration and a considerable waste of time as programmes have to be rewritten. The computer is certainly an expensive addition to the diagnostic tools now available to the cardiologist, yet the initial considerable investment will be amply repaid over the years, for its precise analysis of complex data is unrivalled, its speed of action is unchallenged, and, furthermore, it relieves staff of the tedium of time-consuming routine analyses and mathematical manipulations.

The computer programmes described have become operational owing to the unstinted efforts of Drs. Harry Fozzard, Frank Yanowitz, Plato Kinias, David Wallace, and Dario Domizi.

Fig. I-4 are reproduced from Cox et al. (1969) by kind permission of the authors and editors.

\section{References}

Blackstone, E. (1967). Circulatory Hemodynamics. Úniversity of Chicago Biological Sciences Computation Center, Program No. 000166.

Bondurant, S. (I969). Introduction. Research on acute myocardial infarction. Circulation, 39-40, Suppl. 4, $\mathrm{I}$.

Brodman, K., Erdmann, A. J., Jr., Lorge, I., and Wolff, H. G. (1949). The Cornell Medical Index: an adjunct to medical interview. Fournal of the American Medical Association, 140, 530.

-, Van Woerkom, A. J., Erdmann, A. J., Jr., and Goldstein, L. S. (1959). Interpretation of symptoms with a data-processing machine. Archives of Internal Medicine, 103, 776.

Brown, K. W. G., MacMillan, R. L., Forbath, N., Mel'grano, F., and Scott, J. W. (1963). Coronary unit. An intensive-care centre for acute myocardial infarction. Lancet, 2, 349.

Caceres, C. A. (1963). A basis for observer variation in electrocardiographic interpretation. Progress in Cardiovascular Disease, 5, $52 \mathrm{I}$.

- (1965). Computation for research: a by-product of clinical application. Annals of the New York Academy of Sciences, 126, 926.

-, Steinberg, C. A., Gorman, P. A., Calatayud, J. B., Dobrow, R. J., and Weihrer, A. L. (I964). Computer aids in electrocardiography. Annals of the New York Academy of Sciences, 118, 85.

Coleman, D. J., and Caceres, C. A. (1963). The acoustic vibrations of the heart and computer analysis. In The Theory and Practice of Auscultation, p. 102. Ed. by B. Segal. F. A. Davis, Philadelphia.
Collen, M. F., Rubin, L., Neyman, J., Dantzig, G. B., Baer, R. M., and Siegelaub, A. B. (1964). Automated multiphasic screening and diagnosis. American fournal of Public Health and the Nation's Health, 54, 74I.

Cooper, J. K., and Caceres, C. A. (1964). Transmission of electrocardiograms to computers. Military Medicine, 129, 457.

Cox, J. R., Jr., Fozzard, H. A., Nolle, F. M., and Oliver, G. C. (1969). Some data transformations useful in electrocardiography. In Computers in Biomedical Research, Vol. 3, p. 181. Ed. by R. W. Stacy and B. D. Waxman. Academic Press, New York and London.

Day, H. W. (1963). An intensive coronary care area. Diseases of the Chest, 44, 423.

Dobrow, R. J., Calatayud, J. B., Abraham, S., and Caceres, C. A. (1964). A study of physician variation in heart-sound interpretation. Medical Annals of the District of Columbia, 33, 305.

Frommer, P. L. (I968). The myocardial infarction research program of the National Heart Institute. American fournal of Cardiology, 22, 108.

Gerbarg, D. S., Holcomb, F. W., Jr., Hofler, J. J., Bading, C. E., Schultz, G. L., and Sears, R. E. (I962). Analysis of phonocardiogram by a digital computer. Circulation Research, II, 569.

Konecci, E. B. (1964). In Electronic and ComputerAssisted Studies of Bio-Medical Problems, p. 169. Ed. by O. H. Schmitt and C. A. Caceres. C. C. Thomas, Springfield, Illinois.

Ledley, R. S., and Lusted, L. B. (1959). Reasoning foundations of medical diagnosis. Science, 130, 9.

Lipkin, M., and Hardy, J. D. (1958). Mechanical correlation of data in differential diagnosis of hematological diseases. Fournal of the American Medical Association, 166, I13.

Meltzer, L. E., Palmon, F., Ferrigan, M., Pekover, J., Sauer, H., and Kitchell, J. R. (1964). Prothrombin levels and fatality rates in acute myocardial infarction. Fournal of the American Medical Association, $187,986$.

Nash, F. A. (1954). Differential diagnosis: an apparatus to assist the logical faculties. Lancet, I, 874 .

- (1960). Diagnostic reasoning and the logoscope. Lancet, 2, 1442.

Pipberger, H. V. (1965). Advantages of three lead cardiographic recordings. Annals of the New York Academy of Sciences, 126, 873.

- and Stallmann, F. W. (1964). Computation of differential diagnosis in electrocardiography. Annals of the New York Academy of Sciences, 115, 1115.

- — Y Yano, K., and Draper, H. W. (I963). Digital computer analysis of the normal and abnormal electrocardiogram. Progress in Cardiovascular Disease, 5, 378

Slack, W. V., Hicks, G. P., Reed, C. E., and Van Cura, L. J. (1966). A computer-based medical-history system. New England fournal of Medicine, 274, 194.

- , and Van Cura, L. J. (1968). Patient reaction to computer-based medical interviewing. Computers and Biomedical Research, 1, 527

Schmitt, O. H. (1962). Application of computers in cardiovascular disease. Circulation Research, 2I, 505.

Taylor, S. H. (1966). Measurement of the cardiac output in man. Proceedings of the Royal Society of Medicine, 59, Suppl., 35.

Warner, H. R. (1965). Some computer techniques of value for study of circulation. In Computers in Biomedical Research, Vol. 2, p. 239. Ed. by R. W. Stacy and B. D. Waxman. Academic Press, New York and London. 
Warner, H. R. (1969). Computer-based patient monitoring. In Computers in Bicmedical Research, Vol. 3, p. 239. Ed. by R. W. Stacy and B. D. Waxman. Academic Press, New York and London.

, Swan, H. J. C., Connully, D. C., Tompkins, R. G., and Wood, E. H. (I953). Quantitation of beat-to-beat changes in stroke volume from aortic pulse contour in man. Fourv'al of Applied Physiology, 5,495 .

, Toronto, A. F., Veasey, I. G., and Stephenson,
R. (I96I). A mathematical approach to medical diagnosis. Application to congenital heart disease. fournal of the American Medical Association, 177, I77.

Weid, L. L. (1967). The patient's record as an extension of the basic science training of the physician. Western Reserve University Monograph.

Zadeh, L. A. (1962). From circuit theory to system theory. Proceedings of the Institute of Radio Engineers, 50, 856. 